\title{
Imitation or Early Imitation: Towards the Problem of Primary Data Entry
}

\author{
Igor Val. Danilov \\ Academic Center for Coherent Intelligence (ACCI)
}

\begin{abstract}
The paper highlights the discrepancy between newborns' communication disability and their successful performance in social tasks. The author believes that the "blank mind" can only acquire social meanings by social interaction; it is the only possible way of knowledge acquisition that obeys Nature's laws. There is no other mechanism of transmitting information about social reality to the "blank mind", which can help newborns succeed in imitation. In specific, the article discusses: (1) the terms "early imitation" and "imitation", showing them as two different phenomena of mirror actions in, respectively, newborns and infants (at 8-12 month-olds); (2) the lack of evidence on matching in studies may show that experiments on "early imitation" did not consider emotional arousal as an independent variable of their research; (3) the problem of Primary Data Entry (PDE), how a "blank mind" acquires the first social meanings to initiate knowledge acquisition.
\end{abstract}

Keywords: coherent intelligence, emotions, emotional contagion, imitation, interpersonal synchrony, newborns behavior, primary data entry, social cognition, socialization, theory of mind

\section{INTRODUCTION}

Cognitive, emotional and social development require the ability to categorize and associate the social reality. The growing empirical evidence shows that social cognition is not reducible to the sum of individual cognitive mechanisms and their interactions are something more than a context for social cognition: they can complement and even replace individual mechanisms (De Jaegher et al., 2010). The individual learns behaviors through entrainment, imitation, emotional contagion and interactional synchrony and cannot be therefore dissociated from society during the first months of life (Keromnes et al., 2019). Over four recent decades the phenomenon of imitation in newborns was discussed from two opposite approaches. They are both plausible because the literature contains both empirical results that are not consistent with one another

and data that are consistent but can be interpreted in different ways (Jones, 2009). A noticeably number of studies have posted that neonates are able to imitate a specific range of simple behaviors (Meltzoff 2005). 'There is strong evidence that neonates imitate previously unseen behaviors (Simpson et al., 2014, p. 2)'. From the other hand, growing opposite data proposed that the ability to imitate the behaviors of others is not present at birth, but instead appears much later. There are three main arguments against the phenomenon of imitation in newborns: (a) the imitation in neonates is not well supported by evidence (it is not always replicable) and it does not evidently occur in all experiments (Anisfeld, 2005; Jones, 2009; Jones, 2016; Vincini et al., 2017); (b) the correspondence problem, i.e., it is not clear how newborns could be enough aware of their own movements and how these map onto the movements of others (Jones, 2009; Jones, 
2016); (c) emotional sharing increases infants' actions therefore mirroring an action may be just a byproduct of the action itself (Anisfeld, 2005; Jones, 2009; Vincini et al., 2017) for this reason imitation advocates should agree that tongue protrusion is also an emotional response in addition to being an imitation (Vincini et al., 2017). The latter argument proposes that the pure imitation can be observed when it is separated from emotional arousal. Therefore from these perspectives, this phenomenon is not imitation but a random match between a behavior that the neonates find curious to watch and the behavior that the newborns produce when they are watching something unusual (Jones, 2016).

\section{PROBLEM OF THE TERM “IMITATION" IN NEWBORNS}

The founders of this discussion Meltzoff and Moore (1977) initiated their famous work with the following thought: 'Piaget and other students of developmental psychology consider the imitation of facial gestures to be a landmark achievement in infant development. Infants are thought to pass this milestone at approximately 8 to 12 months of age. Infants younger than this have been postulated to lack the perceptualcognitive sophistication necessary to match a gesture of their own which they cannot see [Piaget,1963] (Meltzoff and Moore, 1977, p.75)'. In this introduction to their findings, the authors defined that the core feature of imitation is "perceptual-cognitive sophistication necessary to match a gesture of their own". And that this infants' ability occurs at the age of 8- to 12-months. The latter standpoint is consistent with empirical data, common sense and modern usage of the term "imitation".

The generally used concept of imitation is defined in various ways, very similar at their core: 'the process of copying the behavior of another person, group, or object, intentionally or unintentionally (APA Dictionary of Psychology, 2020)'. This definition links an observer with social meanings, i.e., an observer is required to categorize social reality by operating with social phenomena (at least with social meanings of self and others, meaning of copying etc.) and therefore can reproduce the topography of both transitive and intransitive actions (Ray and Heyes, 2011).

Growing empirical data and common sense suppose that newborns are not able to understand meanings of social reality on their own in such an extent to be able to categorize social surrounding and to repeat behavior of others on their own (Danilov, 2020c). This could be the reason why in their article the authors also introduced another term of the observed phenomenon calling it "early imitation" (they use it also in subsequents articles Meltzoff and Moore (1983) combining the two concepts). 'This early imitation might be based on an innate releasing mechanism (Meltzoff and Moore, 1977, p. 77)', and 'by our account even this early imitation involves active matching to an environmentally provided target or "model" (Meltzoff and Moore, 1983, p. 708)'. It seems that the context of the usage of these two terms in the articles implied different meanings for the notion of "early imitation" than to the notion of "imitation". It seems that at the moment of establishing the term defining the phenomenon, the authors meant ahead another meaning for the phenomenon itself than the concept of the imitation.

Many researchers, such as Simpson et al. (2014), Jones (2016), emphasized the difference between the observed phenomenon and the term imitation, and that the term imitation should be applied for behaviors that engage awareness of both the goals and means of the pattern (Xavier, J., et al., 2013). At the same time, the term "early imitation" evolved in "neonatal imitation" (Meltzoff and Moore, 1997; Ray and Heyes, 2011; Nagy et al., 2013; Oostenbroek et al., 2016) - i.e., "neonatal imitation" which 'refers to the ability of infants to match others' actions in the first four weeks of life (Simpson et al., 2014, p.3)' - and "newborn imitation" (Jones, 2016). The notion has thus acquired an unusually broad meaning and even in some recent articles, the different terms "early imitation" and "neonate imitation" (or newborn) have been applied to the phenomenon at the same time together. It is important to note that semantically "early imitation" can mean

ancient, and/or initial, and/or prior, and/or primitive, etc etc which do not signify just the pure imitation. In contrast, the term "neonatal imitation" (or newborn) means imitation in an infant who is younger than four weeks old. 


\section{MIRRORING MOVEMENTS ARE CAUSED BY EMOTIONAL AROUSAL}

The term establishes an approach to the study of the phenomenon. The current article highlights the operational definition of the phenomenon and from now on it suggests to use the term "early imitation" because a correct definition of the phenomenon is important for establishing 'the primary empirical question that should be investigated (Vincini et al., 2017, p.3)'. Unfortunately this new notion of "early imitation" is still undefined meanwhile experiments on the phenomenon have been conducted anyway during the last 40 years. The operational definition requires both a semantic competence to differentiate the meaning of a word in modern usage and a correct analysis of relevant empirical data on the modality of this phenomenon. While the appearance of "early imitations" is questioned due to their unstable manifestations in experiments - tongue protrusion is probably only one convincingly reliable finding (Jones, 2016). Evidence that newborns are able to imitate a range of facial gestures seemingly provides the foundation for a weak of the stimulus argument to this conclusion (Chomsky, 1975). Probably, evidence that newborns are able to imitate a range of facial gestures is limited due to the fact that studies on imitation were focused on finding another phenomenon instead of the one they named "early imitation". The discrepancy between the phenomenon and its term seems to provide the foundation for a weak of the stimulus argument to this conclusion (about the ability to mirror actions in newborns), but not an absence of the phenomenon itself. Further research is needed to develop a clear definition. In the meantime the current article highlights the core features and purpose of the phenomenon of "early imitation" which is aimed at the categorization and association of social reality in newborns without their ability to purposely imitate (ability to the 'perceptualcognitive sophistication necessary to match a gesture of their own'). It is possible that current findings indicate only some of the properties of the phenomenon called "early imitation", and future research will obtain more consistent results and show other features of it, applying the proper definition. Finally, the current paper supports the statement that imitation contributes to recognition of the visual similarity at the age of 8- to 12-months. The phenomenon of mirroring movements observed in newborns is a manifestation of another phenomenon with different modality caused by emotional arousal. Newborns are required to begin social interaction even before they would be able to develop their own ability to communicate which will happen later at around 8- to 12 -months. This is because they need to operate with already a minimum set of social meanings before they begin to communicate. Therefore the appearance of such social learning can be seen and should be seen in their behavior from the very beginning. The above arguments pose a problem of primary data entry, the author also believes that the correspondence problem is reducible to this universal problem of primary data entry.

\section{PROBLEM OF PRIMARY DATA ENTRY}

Understanding social reality is essential for individuals to perform immediate reactions and strategic planning in an environment with quickly changing elements (Danilov, in press). Brains provide an evolutionary advantage for survival by allowing individuals to extract modules of information that bear predictions (Prochazkova and Kret, 2017; Adolphs, 2001). The latter is grounded on the ability of capturing mental states of other individuals promoting social cooperation and competition (Danilov, 2020b). The appearance of communication requires a shared understanding of the signal's meaning (i.e., uses) within a particular context among a community of users (Wittgenstein, 1973). Acquisition of knowledge implies some initial data, because new knowledge can be assimilated based on the discovery of new key relationships between cause and effect within the previous knowledge, and/or opening links between elements of initial knowledge and new information domains. This means that primary knowledge is crucial for the initial stages of cognitive development and even for assimilation of simple social tasks. On the other side at the initial stages of the development infants are not able to communicate with caregivers effectively enough to acquire first social phenomena on their own (there is no evidence of any communication at initial stages). At the same time they are able anyway to successfully perform their social tasks and assimilate social knowledge on their own. Categorization of social reality by newborns requires primary data entry, considering (i) the minimum set of social knowledge needed from the birth to begin cognitive development, 
and (ii) the presence of thousands different linguistic communities with their specific social reality. (Danilov and Mihailova, 2019). The problem of PDE can be divided into three sub-questions:

(Q1) The reality represents to the mind an infinite amount of objects which can be reduced to only one phenomena just in a thought experiment. In reality, the mind relates at least decades of phenomena which appear to the mind at the same time through many stimuli. How can the "blank mind" distinguish different stimuli to properly combine them in relation to different objects?

(Q2) How can a newborn take into account only one phenomenon from reality, distinguishing it from others if he/she doesn't know no ones? How can a newborn understand that reality is a compound of phenomena and not a united and unique entity? The ability to intuit the only one phenomenon from many others needs an ability to categorize reality, which needs initial knowledge on it.

(Q3) The meaning of the phenomenon can differ in different communities. While maintaining the stability of the phenomenon's meaning within the community is vital for the stable existence of the phenomena and communication within community. How can the "blank mind" highlight different phenomena with certain meanings which are similar with its general meaning in these community.

This PDE problem challenges our knowledge on modalities of social interaction.

\section{DISCUSSION}

There are a few comments for a discussion about early imitation:

a) Imitation is the ground of associative learning in infants, where visual input is probably the core. At the same time, it is widely argued that language and emotional expression in infants with congenital Visual impairment (VI) is not delayed from their unimpaired peers at the first year of life (Danilov, 2020a). Infants with VI are able to associate first words at the period of neurological development which is not significantly different with their non-disabled peers (Perez-Pereira and Conti-Ramsden, 1999). Among children with VI, the initial stages of their mental development follow the same trajectory as in unimpaired children.

b) Newborns also demonstrate other social achievements: such as preference of faces and even race, which are not consistent with their cognitive and communicative abilities

c) The mentioned above discrepancy in results (the lack of evidence on matching) is probably because studies on "early imitation" did not consider emotional arousal as an independent variable of their research. If studies conducted their experiments under two different conditions comparing their results with the baseline without emotional arousal, they would have probably obtained more consistent outcomes.

\section{CONCLUSIONS}

The opinion article discusses the terms "imitation" and "early imitation" studying cognitive development in organisms at the Stages 0-3 (Model of hierarchical complexity by Commons) or Sensorimotor (Stage of Cognitive development by Piaget). The paper highlights the problem of the discrepancy between newborns' communication disability and their successful performance in social tasks. The author believes that the "blank mind" can only acquire social meanings by social interaction; it is the only possible way of knowledge assimilation that obeys laws of Nature (Danilov, 2020c). There is no any other mechanism of transmitting information on social reality to the "blank mind" which can help newborns succeed in imitation. The article shows that:

i. There are two different phenomena of mirror actions in newborns and infants: early imitation (in newborns) and imitation (in infants at 8-12 month-olds). Imitation appears at approximately 8 to 12 months (Piaget, 1963; Jones, 2016; Vincini et al., 2017). 'Infants younger than this have been postulated to lack the perceptual-cognitive sophistication necessary to match a gesture of their own which they cannot see [Piaget,1963] (Meltzoff and Moore, 1977, p.75)' The paper suggests intending mirror actions in newborns as "early imitation" (Meltzoff and Moore, 1977) because these actions are entirely different processes from imitation. Early imitation is aimed 
at the categorization and association of social reality in newborns without their ability to purposely imitate, ability to the 'perceptual-cognitive sophistication necessary to match a gesture of their own (Meltzoff and Moore,1977)'.

ii. This new notion of early imitation is still undefined meanwhile experiments on the phenomenon have been conducted anyway during the last 40 years. The author believes that researchers obtained different results due to the impurity of the term's definition. The operational definition requires both a semantic competence to differentiate the meaning of a word in modern usage and a correct analysis of relevant empirical data on the modality of this phenomenon.

iii. More research needs to define this phenomenon of early imitation. It is possible that current findings indicate only some of the properties of the phenomenon called early imitation, and future research will obtain more consistent results and show other features of it, applying the proper definition.

iv. There is a new direction for the research on early imitation in newborns. The mentioned above discrepancy in results (the lack of evidence on matching) is probably because studies on "early imitation" did not consider emotional arousal as an independent variable of their research. If studies conducted their experiments under two different conditions comparing their results with the baseline without emotional arousal, they would have probably obtained more consistent outcomes.

v. The phenomenon of early imitation highlights the problem of Primary Data Entry (PDE): how a "blank mind" acquires the first social meanings to initiate knowledge acquisition.

\section{REFERENCES}

Adolphs, R. (2001). The neurobiology of social cognition. Curr. Opin. Neurobiol, 11(2), 231-239. Anisfeld, M. (2005). No compelling evidence to dispute Piaget's timetable of the development of representational imitation in infancy. In S. Hurley \& N. Chater (Eds.), Perspectives on imitation: From neuroscience to social science, 2: Imitation, human development, and culture (pp. 107-131). Cambridge, MA: MIT Press.

APA Dictionary of Psychology. (2020). Retrieved June 2, 2020, from https://dictionary.apa.org

Arabin B., Bos R., Rijlaarsdam R., Mohnhaupt A., \& van Eyck J. (1996) The onset of inter-human contacts: Longitudinal ultrasound observations in early twin pregnancies. Ultrasound Obstet Gynecol, 8(3), 166-73.

Danilov, I.V., \& Mihailova, S. (2019). Social interaction shapes infants' earliest links between language and cognition. Social Sciences Bulletin, 2(29), 145-157. ISSN 1691-1881, eISSN 2592-8562

Danilov, I.V. (2020a). Emotions in learning towards coherent intelligence: The review of studies on social behavior in infants with visual impairment. J Med - Clin Res \& Rev., 4(4), 1-6.

Danilov, I.V. (2020b). Ontogenesis of Social Interaction: Review of Studies Relevant to the Fetal Social Behavior. J Med - Clin Res \& Rev., 4(2), 1-7.

Danilov, I.V. (2020c). Social Interaction in Knowledge Acquisition: Advanced Curriculum. Critical Review of Studies Relevant to Social Behavior of Infants. The Twelfth International Conference on Advanced Cognitive Technologies and Applications COGNITIVE 2020. DOI: https://doi.org/10.33423/jhetp.v20i12

De Jaegher, H., Di Paolo, E., \& Gallagher, S. (2010). Can social interaction constitute social cognition? Trends in Cognitive Sciences, 14(10). doi:10.1016/j.tics.2010.06.009

Jones, S. (2009). The development of imitation in infancy. Phil. Trans. R. Soc. B, 364, 2325-2335. doi:10.1098/rstb.2009.0045

Jones, S. (2016). Can newborn infants imitate? Cogn Sci. doi: 10.1002/wcs. 1410

Keromnes, G., Chokron, S., Celume, M-P., Berthoz, A., Botbol, M., Canitano, R., . . . Tordjman, S. (2019). Exploring Self-Consciousness From Self- and Other-Image Recognition in the Mirror: Concepts and Evaluation. Front. Psychol., 10, 719. doi: 10.3389/fpsyg.2019.00719 
Meltzoff, A.N., \& Moore, M.K. (1977). Imitation of facial and manual gestures by human neonates. Science, 198, 75-78. doi: 10.1126/science.198.4312.75

Meltzoff, A.N., \& Moore, M.K. (1983). Newborn infants imitate adult facial gestures. Child Development, 54, 702-709.

Meltzoff, A.N. (2005). Imitation and other minds: the 'like me' hypothesis. In S. Hurley \& N. Chater (Eds.), Perspectives on imitation: From neuroscience to social science, 2 (pp. 55-78). Cambridge, MA: MIT Press.

Nagy, E., Pilling, K., Orvos, H., \& Molnar, P. (2013). Imitation of tongue protrusion in human neonates: Specificity of the response in a large sample. Dev. Psychol., 49, 1628-1638. doi: $10.1037 / \mathrm{a} 0031127$

Oostenbroek, J., Suddendorf, T., Nielsen, M., Redshaw, J., Kennedy-Costantini, S., Davis, J., . . Slaughter, V. (2016). Comprehensive longitudinal study challenges the existence of neonatal imitation in humans. Curr. Biol., 26, 1334-1338. doi: 10.1016/j.cub.2016.03.047

Perez-Pereira, M., \& Conti-Ramsden, G. (1999). Language Development and Social Interaction in Blind Children. Retrieved March 1, 2020, from https://books.google.it/books?id=9wd1xXuYwmwC

Prochazkova, E., \& Kret, M.E. (2017). Connecting minds and sharing emotions through mimicry: A neurocognitive model of emotional contagion. Neuroscience and Biobehavioral Reviews, 80, 99_ 114. http://dx.doi.org/10.1016/j.neubiorev.2017.05.013.

Ray, E., \& Heyes, C. (2011). Imitation in infancy: The wealth of the stimulus. Developmental Science, 14(1), 92-105. DOI: 10.1111/j.1467-7687.2010.00961.x

Simpson, E.A., Murray, L., Paukner, A., \& Ferrari, P.F. (2014). The mirror neuron system as revealed through neonatal imitation: Presence from birth, predictive power and evidence of plasticity. Philos. Trans. R. Soc. Lond. B Biol. Sci., 369, 20130289. doi: 10.1098/rstb.2013. 0289.

Vincini, S., Jhang, Y., Buder, E.H., \& Gallagher, S. (2017). Neonatal Imitation: Theory, Experimental Design, and Significance for the Field of Social Cognition. Front. Psychol., 8(1323). doi: 10.3389/fpsyg.2017.01323

Wittgenstein, L. (1973). Philosophical investigations. Prentice Hall.

Xavier, J., Tilmont, E., \& Bonnot, O. (2013). Children's synchrony and rhythmicity in imitation of peers: Toward a developmental model of empathy. J. Physiol. http://dx.doi.org/10.1016/j.jphysparis.2013.03.012 